\title{
Tubercular Mycotic Aortic Aneurysm: Complication of Tubercular Spondylodiscitis due to Treatment Default
}

\author{
Neeraj Kumar ${ }^{1}$ Mini Sharma ${ }^{1}$ Nishant Nayyar ${ }^{1}$ \\ ${ }^{1}$ Department of Radiodiagnosis, Dr. Rajender Prasad Government \\ Medical College, Tanda, Kangra, Himachal Pradesh, India \\ 2 Department of Radiodiagnosis, Dr. Rajender Prasad Government \\ Medical College, Tanda, Kangra, Himachal Pradesh, India
}

\author{
Lokesh Rana² Dinesh Sood ${ }^{2}$
}

Int J Recent Surg Med Sci 2022;8:114-117.

\author{
Address for correspondence Neeraj Kumar, MD, Department of \\ Radiodiagnosis, Dr. Rajender Prasad Government Medical College, \\ Tanda, Kangra, Himachal Pradesh 176001, India \\ (e-mail: dr.neerajchaudhary525@gmail.com).
}

\begin{abstract}
Keywords

- cardiovascular tuberculosis

- mycotic aneurysm

- spinal tuberculosis

- thoracic aortic aneurysm

- tuberculosis

We have reported rare case of tubercular aortic aneurysm which has developed as complication of spinal tuberculosis. Imaging of patient is available prior to starting of antitubercular treatment which showed vertebral tuberculosis with absence of aortic aneurysm. However, patient defaulted on treatment after taking antitubercular chemotherapy only for 2 months. Patient again reported after 14 months with chest pain. At this stage, imaging showed progression of spinal tuberculosis with development of pseudoaneurysm in adjacent descending thoracic aorta. This contiguous spread of tuberculosis from spine to aorta may have been prevented if patient had completed complete course of antitubercular therapy with regular follow-ups. Patient of spinal tuberculosis need to be counselled and informed regarding its various complications, so that they remain adhered to antitubercular chemotherapy and timely follow-ups to prevent complications.
\end{abstract}

\section{Introduction}

Descending thoracic aortic aneurysm due to contiguous spread from spinal tuberculosis is a rare disease with high morbidity and mortality. ${ }^{1,2}$ First case of tubercular aortic aneurysm was reported in 1895 by Kamen. ${ }^{3}$ Patient may have variable presentation ranging from asymptomatic state, constitutional symptoms, chest pain to hypovolemic shock, and death. Early diagnosis can significantly improve patient outcome. Tuberculous aortic aneurysm occurring secondary to contiguous spread from lung or vertebral tuberculosis can be prevented by timely management of these infections.

\section{Case Report}

A 39-year-old male had presented with history of back pain for 3 months and weakness of bilateral lower limbs with bladder and bowel incontinence. Erythrocyte sedimentation rate (ESR) was raised $(76 \mathrm{~mm}$ ) and PCR (polymerase chain reaction) was positive for mycobacterium tuberculosis DNA (deoxyribose nucleic acid). The Mantoux test revealed induration of $17 \mathrm{~mm}$. Renal and liver function tests were normal. Chest X-ray showed mediastinal widening which had lobulated margins on right side (-Fig. 1A). Magnetic resonance imaging (MRI) thoracic spine (- Fig. 1) showed altered signal intensity involving D4-8 vertebrae showing enhancement
DOI https://doi.org/ 10.1055/s-0041-1740504. ISSN 2455-7420.

\footnotetext{
(c) 2022. Medical and Surgical Update Society. All rights reserved. This is an open access article published by Thieme under the terms of the Creative Commons Attribution-NonDerivative-NonCommercial-License, permitting copying and reproduction so long as the original work is given appropriate credit. Contents may not be used for commercial purposes, or adapted, remixed, transformed or built upon. (https://creativecommons.org/ licenses/by-nc-nd/4.0/)

Thieme Medical and Scientific Publishers Pvt. Ltd., A-12, 2nd Floor, Sector 2, Noida-201301 UP, India
} 

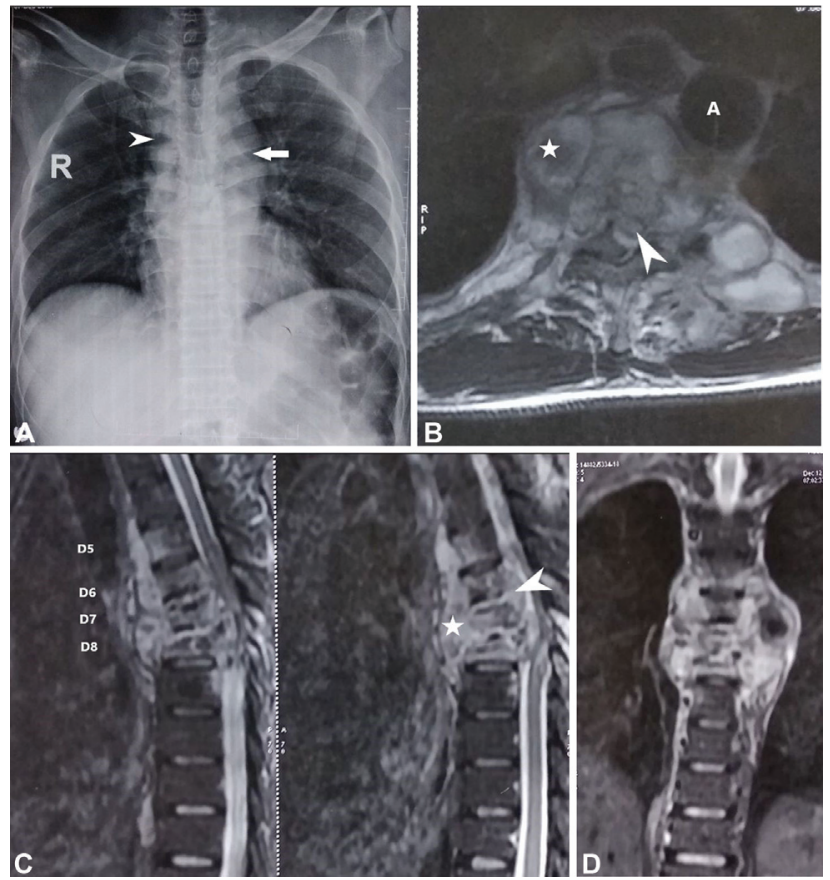

Fig. 1 (A) Chest X-ray (posteroanterior view) showing mediastinal widening (white arrow) having lobulated margins on right side (white arrowhead). (B) Axial T2W (C) Sagittal and (D) coronal T1W post contrast MRI showing altered signal intensity involving D5-D8 vertebrae with erosion destruction of D7 and D8 vertebrae with paravertebral soft tissue (white star) extending into epidural space (white arrowhead) leading to spinal canal space compromise with compressive myelopathy. Note can be made of normal calibre of visualised adjacent descending thoracic aorta (A). MRI, magnetic resonance imaging; $W$, weighted.

on postcontrast images. Heterogeneously enhancing paravertebral soft tissue was noted with epidural extension leading to spinal canal space compromise. Patient was started on antitubercular therapy with rifampin, isoniazid, ethambutol, pyrazinamide, and streptomycin. However, patient defaulted after taking this chemotherapy only for 2 months and lost to follow-up.

Patient again presented to our institute after 14 months with severe chest pain. Chest radiograph showed widening of superior mediastinum which had lobulated outline on right side and well-defined sharp margin on left side having sharp interface with adjacent lung and was silhouetting with aortic knuckle. This mediastinal widening has markedly increased as compared with previous chest X-ray. No shifting of trachea was noted. Contrast-enhanced computed tomography (CECT; - Fig. 2) showed saccular pseudoaneurysm arising from descending thoracic aorta measuring $7.7 \times 9.4 \times 10.3 \mathrm{~cm}$ (anteroposterior $\times$ transverse $\times$ craniocaudal) showing eccentric thrombus with maximum thickness of $5.1 \mathrm{~cm}$ on right side. Neck of aneurysm measures $1.9 \mathrm{~cm}$. There was erosion destruction of adjacent vertebral bodies with widening of left neural foramina. There was also peripherally enhancing paravertebral soft tissue from D4-8 level with epidural extension leading to spinal canal space compromise. On MRI of dorsal spine ( $\mathbf{F i g . 2 E , ~ F ) , ~ p a r a v e r t e b r a l ~ a b s c e s s ~ w a s ~ h y p e r i n t e n s e ~ o n ~}$ T2-weighted image (WI) with maximum thickness of $24.3 \mathrm{~mm}$ on right side and had caused widening of left neural foramen with epidural extension. Pseudoaneurysm of adjacent descending thoracic aorta showed flow related signal loss and thrombosed part was heterogeneously hyperintense. Endovascular treatment was ruled out by cardiothoracic and vascular surgeon (CTVS) due to involvement of longer segment of aorta. Moreover, he was found unfit for cardiac anesthesia during preanesthetic consultation. Patient and family members opted to continue with conservative management with antitubercular therapy. However, patient died after 4 months on follow-up.

\section{Discussion}

Aortic aneurysm is pathologic dilatation or ballooning of aortic segment that has the tendency to expand and rupture. ${ }^{4}$ Quantitatively, it is defined as diameter exceeding 1.5 times of the normal values for the same aortic segment in unaffected individuals of same age and sex. ${ }^{5}$ Mycotic aneurysm is infectious (can be bacterial, viral, or fungal in origin) break in the wall of artery with formation of a blind, saccular outpouching which is contiguous with the arterial lumen. ${ }^{6}$ "Mycotic aneurysm" is a misnomer for infected aneurysm coined by Osler in $1885 .^{7}$ The term "mycotic" denotes mushroom-like appearance of aneurysm rather than underlying microbial etiology. ${ }^{8}$ Mycotic aneurysms commonly affect major arteries at their branch points. The femoral artery is most frequently involved, followed by the abdominal, thoracoabdominal, and thoracic aortas. ${ }^{8}$ Aneurysms of peripheral, intracranial, and visceral arteries are rare. ${ }^{6}$ The prevalence of infected aortic aneurysms is 0.7 to $1 \%$ of all surgically treated aortic aneurysms. ${ }^{9}$ Thoracic aorta aneurysms are classified into ascending aortic aneurysms which are most common $(\approx 60 \%)$, followed by aneurysms of the descending aorta $(\approx 35 \%)$ and aortic arch $(<10 \%){ }^{10}$ Most common pathogen causing mycotic aortic aneurysm are Staphylococcus aureus (28\%), Salmonella spp. (15\%), and Pseudomonas aeruginosa (10\%) in Western countries. ${ }^{9}$ However, Salmonella is the most common pathogen reported in Asian counties. ${ }^{11}$

Spinal tuberculosis leading to aneurysm of descending thoracic aorta is a rare complication. Following four mechanisms have been described for involvement of aortic wall in tuberculosis: (1) direct implantation of tuberculous bacilli over intima of aortic wall, presence of atherosclerotic patch predisposes for direct implantation; (2) hematogenous spread carried to adventitia or media by vasa vasorum; (3) involvement of aortic wall indirectly via lymphatics; and (4) contiguous spread from adjacent lymph node, pulmonary focus, abscess, or bone. ${ }^{3}$

Probable cause of aortic involvement in our case was contiguous spread from spinal tuberculosis, as patient was already diagnosed with spinal tuberculosis and had defaulted on antitubercular chemotherapy. Contiguous spread is also the most common cause of tubercular aortic aneurysm. ${ }^{12,13}$ Clinically tubercular aortic pseudoaneurysm may be asymptomatic or it may present with chest pain, abdominal pain, back pain, features of hypovolemic shock, or hemorrhage into lung or gastrointestinal tract. 

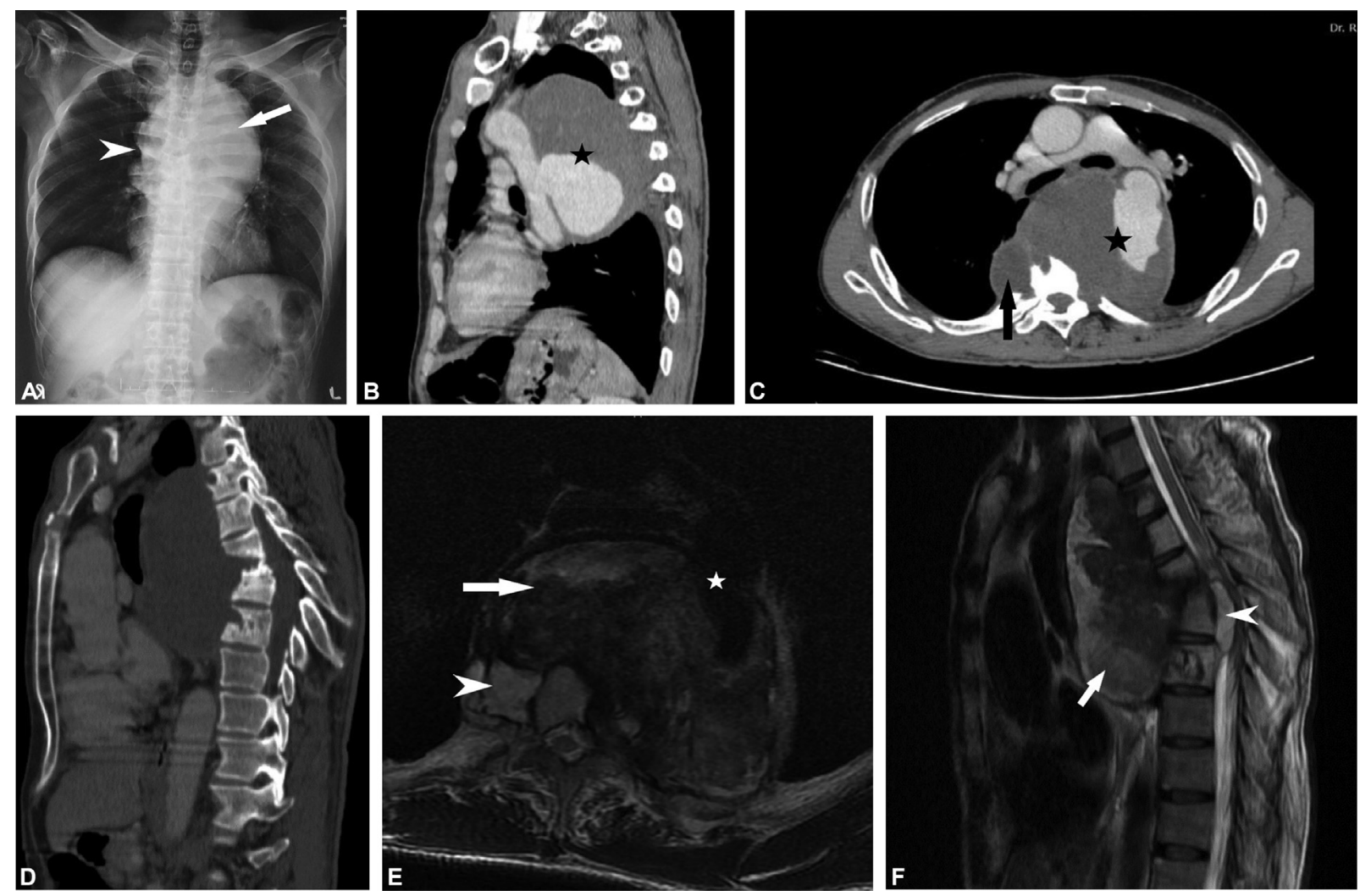

Fig. 2 Follow up imaging after 14 months. (A) Chest X-ray (posteroanterior view) showing increased superior mediastinal widening (white arrow) as compared to initial X-ray with lobulated margin on right side (white arrowhead). (B), (C) Contrast enhanced computed tomography (CECT) sagittal and axial images showing partially thrombosed aneurysm of descending thoracic aorta (black star) with paravertebral abscess (black arrow). (D) Sagittal CECT bone window images show erosion and sclerosis of D4-D8 vertebrae. (E) Axial T2W MRI shows flow void in descending thoracic aorta and patent part of aneurysm (white star). Thrombosed part is heterogeneously hyperintense (white arrow). Right sided paravertebral abscess is also seen (white arrowhead). (F) Sagittal T2W MRI shows extent of aortic aneurysm (white arrow), erosion and altered signal intensity involving D4-D8 vertebrae and epidural part of paravertebral abscess posterior to D7 vertebral body (white arrowhead). MRI, magnetic resonance imaging; W, weighted.

Treatment of tubercular pseudoaneurysm of aorta includes surgical management along with antitubercular chemotherapy. ${ }^{14}$ Surgical treatments include endovascular stenting, endovascular aneurysm repair, and anatomical bypass. Anatomical bypass includes resection of diseased segment and reconstruction of distal vessel using graft from noninfected region. ${ }^{15}$ In our case, if patient had consented, endovascular stent-graft under cover of antitubercular therapy would have been treatment of choice.

\section{Conclusion}

In summary, we have reported a case of 39-year-old male who had developed spinal tuberculosis and was started on antitubercular therapy but defaulted on treatment. Due to which spinal tuberculosis further progressed involving adjacent descending thoracic aorta leading to its pseudoaneurysm. This is probably the first reported case of tubercular aortic pseudoaneurysm in which imaging of patient are available before development of aneurysm. It may be inferred that development of potentially fatal complication like aortic pseudoaneurysm of spinal tuberculosis had developed in this patient due to default of antitubercular chemotherapy. It highlights the need for patient counselling, so that he/she remain adhered to complete course of antitubercular chemotherapy. Moreover, untreated/unrecognized thoracic aortic aneurysms may rupture, possibly with lethal consequences.

Note

The research was carried out at Dr. Rajender Prasad Government Medical College, Tanda, Kangra, Himachal Pradesh, India.

\section{Funding}

None.

Conflict of Interest

None declared.

\section{References}

1 Kolhari VB, Bhairappa S, Prasad NM, Manjunath CN. Tuberculosis: still an enigma. Presenting as mycotic aneurysm of aorta. BMJ Case Rep 2013;2013(01):2013008869

2 Shahabuddin S, Sami SA. Tuberculous ascending aortic pseudoaneurysm. Interact Cardiovasc Thorac Surg 2013;17(04):742-743

3 Xue J, Yao Y, Liu L. Treatment of tuberculous aortic pseudoaneurysm associated with vertebral tuberculosis: a case series 
and a literature review. Medicine (Baltimore) 2018;97(15): e0382

4 Mathur A, Mohan V, Ameta D, Gaurav B, Haranahalli P. Aortic aneurysm. J Transl Int Med 2016;4(01):35-41

5 Harris C, Croce B, Cao C. Thoracic aortic aneurysm. Ann Cardiothorac Surg 2016;5(04):407-407

6 Lee W-K, Mossop PJ, Little AF, et al. Infected (mycotic) aneurysms: spectrum of imaging appearances and management. Radiographics 2008;28(07):1853-1868

7 Kim Y-W. Infected aneurysm: current management. Ann Vasc Dis 2010;3(01):7-15

8 Fisk M, Peck LF, Miyagi K, et al. Mycotic aneurysms: a case report, clinical review and novel imaging strategy. QJM 2012;105(02): 181-188

9 Majeed H, Ahmad F. Mycotic aneurysm. In: StatPearls. Treasure Islane, FL: StatPearls Publishing; 2021
10 Kuzmik GA, Sang AX, Elefteriades JA. Natural history of thoracic aortic aneurysms. J Vasc Surg 2012;56(02):565-571

11 Huang Y-K, Chen C-L, Lu M-S, et al. Clinical, microbiologic, and outcome analysis of mycotic aortic aneurysm: the role of endovascular repair. Surg Infect (Larchmt) 2014;15(03): 290-298

12 Kumar S, Babu NS, Jaret P, Sharma A. Tubercular mycotic aortic aneurysm: a case report. Lung India 2016;33(02):192-195

13 Kouchoukos NT. Tuberculous aneurysm of the aorta: rare but treatable. J Thorac Cardiovasc Surg 2016;152(04):e98-e99

14 Choudhary SK, Bhan A, Talwar S, Goyal M, Sharma S, Venugopal P. Tubercular pseudoaneurysms of aorta. Ann Thorac Surg 2001;72 (04):1239-1244

15 Madabhavi I, Sarkar M, Chauhan C, Modi M. Proximal descending thoracic aortic pseudoaneurysm secondary to Pott's spine. Aorta (Stamford) 2020;8(02):35-37 